\title{
Optical Circuits Based on Polariton Neurons in Semiconductor Microcavities
}

\author{
T. C. H. Liew, ${ }^{1,2}$ A. V. Kavokin, ${ }^{1,3}$ and I. A. Shelykh ${ }^{2,4}$ \\ ${ }^{1}$ School of Physics and Astronomy, University of Southampton, Highfield, Southampton SO17 1BJ, United Kingdom \\ ${ }^{2}$ International Center for Condensed Matter Physics, Universidade de Brasilia, 70904-970, Brasilia-DF, Brazil \\ ${ }^{3}$ University of Rome II, 1, via della Ricerca Scientifica, Rome, 00133, Italy \\ ${ }^{4}$ Science Department, University of Iceland, Dunhaga 3, IS-107, Reykjavik, Iceland \\ (Received 20 March 2008; revised manuscript received 6 May 2008; published 1 July 2008)
}

\begin{abstract}
By exploiting the polarization multistability of polaritons, we show that polarized signals can be conducted in the plane of a semiconductor microcavity along controlled channels or "neurons." Furthermore, because of the interaction of polaritons with opposite spins it is possible to realize binary logic gates operating on the polarization degree of freedom. Multiple gates can be integrated together to form an optical circuit contained in a single semiconductor microcavity.
\end{abstract}

PACS numbers: 71.36.+c, 42.55.Sa, 42.65.Pc

Introduction. - Recently much research has been devoted to the question of whether optical digital processing could offer higher performance than electronic digital processing. Indeed the use of optical waveguides and fibers has allowed the low-loss propagation of signals over extreme distances and the high frequency of light has allowed the generation of ultrashort pulses. However, at the microprocessor level, optics cannot currently meet the low cost and power consumption of modern electronic devices that have benefitted from decades of experience.

Both optical and electronic processing systems require the Coulomb interaction of electrons, whether it is the switching of currents by applied voltages or the changes of refractive index due to nonlinear effects on electron motion. Unfortunately the nonlinear coefficients in materials are generally small, which causes many designs for the all-important optical gates (which provide the processing power of an optical device) to require high powers to function. While some advancements have been made recently using microring resonators [1], we focus our attention on semiconductor microcavities: a system governed neither purely by photons or electrons but rather a mixture of both.

A semiconductor microcavity $[2,3]$ is a planar layered nanostructure consisting of one or more quantum wells sandwiched between a pair of distributed Bragg mirrors. The strong coupling of quantum well excitons with cavity photons results in new quasiparticles known as excitonpolaritons [4] (or polaritons for short). Polaritons demonstrate both a long coherence length [5] due to their photonic component and strong nonlinearity due to Coulomb interactions between excitons [6]. The properties of polaritons have allowed the construction of micron-sized parametric oscillators [7,8], polariton lasers [9-11], and the observation of Bose-Einstein condensation at high temperatures [12-16]. Current research is focused on the electrical injection of polaritons for light emitting devices [17] and polariton superfluidity [18].

Polaritons also have a unique spin structure and spin dynamics [19]; the control of propagating polariton spins has now been evidenced [20] and research has begun to focus on individual spin optoelectronic devices [21,22]. While polaritons directly couple to external light fields allowing their linking to other optoelectronic devices, a significant achievement would be the integration of several elements together in a single microcavity plane. Indeed such integration of functional elements is not an easy task even for conventional optical circuits based on embedding chains of defects in photonic crystals [23].

We demonstrate a technique for making binary logic gates that act on the polarization degree of freedom of polaritons. Different logic gates can be linked together, in the plane of the microcavity, as signals can be carried along controlled channels that are created by patterning the microcavity structure such that polaritons experience a structured potential. In fact, polaritons themselves do not move the whole distance from one end of the channel to the other; rather it is the motion of a circularly polarized domain wall or switching of successive parts of the channel caused by very short propagation of polaritons that results in a long signal propagation. In this sense the channel bears a loose analogy to biological neurons, which is why we call these channels "polariton neurons." Since we do not rely on single polaritons traveling the full length of the channels, the short lifetime of polaritons does not limit the length of signal propagation. The logic gates that we propose rely on the polarization multistability [24] of polaritons in microcavities.

Polariton neurons. - Consider a semiconductor microcavity that is patterned such that polaritons experience the potential shown in Fig. 1(b). This can be achieved by variation of the cavity width [25], applying stress [26], or putting metals on the surface of the cavity [14]. Because of their long coherence time [27] and the fact that in the low density limit they behave as weakly interacting bosons [12], polariton dynamics can be treated in the framework of the mean-field approximation, which leads to the GrossPitaevskii (GP) equation [28,29]. This equation, commonly used in the description of atomic Bose-Einstein condensates [30-32], has been applied to semiconductor 


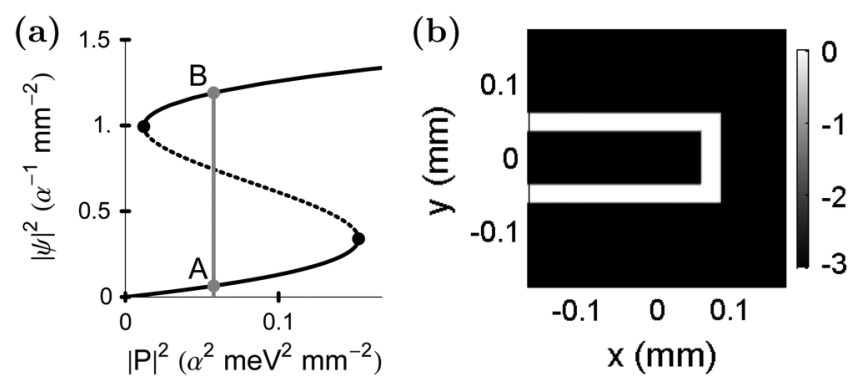

FIG. 1. (a) Dependence of the intensity of a single uncoupled (spin-polarized) polariton state on pump power. The $S$-shaped curve is characteristic of a bistable system [42-44] and it is well known that the middle branch (dotted part of curve) is unstable; that is, the polariton intensity can have a maximum of two possible values, $A$ and $B$, for a given pump intensity. Parameters: $E_{p}-E_{0}=1 \mathrm{meV}, \tau=3$ ps. (b) Polariton potential profile in real space.

microcavities to describe several phenomena, including the suppression of Rayleigh scattering by impurities [29], the spatial structure of microcavity parametric oscillator polaritons [33,34], the dispersion of polariton superfluids [28], polariton condensation in disordered media [35], and the interference of polariton condensates [22].

Polaritons have two possible spin projections on the structure growth axis, $\sigma= \pm 1$, corresponding to the right $\left(\sigma_{+}\right)$and left $\left(\sigma_{-}\right)$circular polarizations of external photons [19]. The spin dependent GP equation is [24]

$$
\begin{aligned}
i \hbar \frac{\partial \psi_{\sigma}}{\partial t}= & \left(\hat{H}_{L P}(-i \hat{\boldsymbol{\nabla}})-\frac{i \hbar}{2 \tau}+W(\mathbf{r})\right) \psi_{\sigma} \\
& +\left(\left|\psi_{\sigma}\right|^{2}+\frac{\alpha_{2}}{\alpha_{1}}\left|\psi_{-\sigma}\right|^{2}\right) \psi_{\sigma}+p_{\sigma}(\mathbf{r}, t) e^{-i E_{p} t / \hbar},
\end{aligned}
$$

where the $\sigma$ polarized internal cavity polariton field $\psi_{\sigma}$ depends on the spatial coordinate $\mathbf{r}$. The kinetic energy operator $\hat{H}_{L P}$ represents the dispersion of polaritons. We consider only lower branch polaritons from the strong light-matter coupling - upper branch polaritons will not be excited under the conditions we propose. $\tau$ is the polariton lifetime. $W(\mathbf{r})$ represents a potential experienced by polaritons. $\alpha_{1(2)}$ is the matrix element of polaritonpolariton interaction in the parallel spin (antiparallel spin) configuration, respectively. It is well known that for 2D excitons and exciton-polaritons the exchange interaction strongly dominates over the direct one, and thus polariton-polariton interactions are anisotropic $\left|\alpha_{2}\right|<\alpha_{1}$ [36-39]. This anisotropy strongly affects the properties of polariton systems in the superfluid regime $[28,40]$ and leads to remarkable effects in polariton spin relaxation, such as self-induced Larmor precession and inversion of the linear polarization during the scattering act [19,39,41]. In Eq. (1) the fields were rescaled so that only the ratio of $\alpha_{2}$ to $\alpha_{1}$ is significant.

The driving optical pump field is given by $p_{\sigma}(\mathbf{r}, t)$ and $E_{p}$ is the pump energy. If the pump energy is tuned greater than $\hbar \sqrt{3} / \tau$ above the polariton eigenenergy (bare polariton branch energy), then for some excitation powers the system can exhibit more than one stable state [42-44]. This can cause intricate effects on parametric oscillators involving the coupling between more than one state $[42,45]$. The dependence of the polariton intensity of a single state in space can be calculated analytically [24] from the GP equation in the stationary regime, if coupling to other points in space is ignored (i.e., one assumes an infinite polariton effective mass):

$$
\left[\left(E_{0}-E_{p}+\left|\psi_{\sigma}\right|^{2}+\frac{\alpha_{2}}{\alpha_{1}}\left|\psi_{-\sigma}\right|^{2}\right)^{2}+\frac{\hbar^{2}}{4 \tau^{2}}\right]\left|\psi_{\sigma}\right|^{2}=\left|p_{\sigma}\right|^{2}
$$

where $E_{0}$ is the bare polariton eigenenergy. If, for simplicity, we consider the excitation of the system by circularly polarized light, then all polaritons will have the same spin and the polariton intensity exhibits an $S$-shaped curve, which characterizes a bistable system [Fig. 1(a)]. If the pump intensity is increased from zero, the polariton intensity increases steadily from zero until the first turning point. For higher pump intensities, the polariton intensity jumps to the upper branch of the $S$-shaped curve. If the pump intensity is then decreased, the polariton intensity remains on this branch, provided the pump intensity is greater than that of the second turning point.

We solve Eq. (1) numerically (with finite effective mass) to model the excitation of the system in Fig. 1(b) with a broad, $\sigma_{+}$polarized, Gaussian, cw pump. To evaluate the kinetic energy term [first term in the right-hand side of Eq. (1)] we use Fourier transforms; it is straightforward to represent the kinetic energy operator in reciprocal space, even with a nonparabolic dispersion. The pump is tuned above the polariton eigenenergy of the channel region and has weak intensity such that polariton intensities lie on the lower branch of the $S$-shaped curve. Because of the larger pump-eigenenergy detuning, hardly any polaritons are excited in the region outside the channel. We then calculate the evolution of the polariton fields after a ( $\sigma_{+}$polarized) pulse is applied near one end of the channel, which locally switches the polariton intensity to the upper branch of the $S$-shaped curve.

The results (Fig. 2) show that after the pulse has decayed, successive regions of the channel switch to the high intensity state. This can be attributed to the kinetic term in Eq. (1), which prefers to smooth the polariton distribution in space [46]; a low intensity region next to a high intensity region tends to grow to a similar intensity, thus also switching to the upper branch of the hysteresis curve. The propagation of the signal continues around corners in the channel, allowing a way of creating wires for optical circuits in the microcavity plane. We note that in principle the containment of polaritons in channels could also be achieved by patterning the weak driving $\mathrm{cw}$ field instead of structuring the potential. The switching mechanism is 


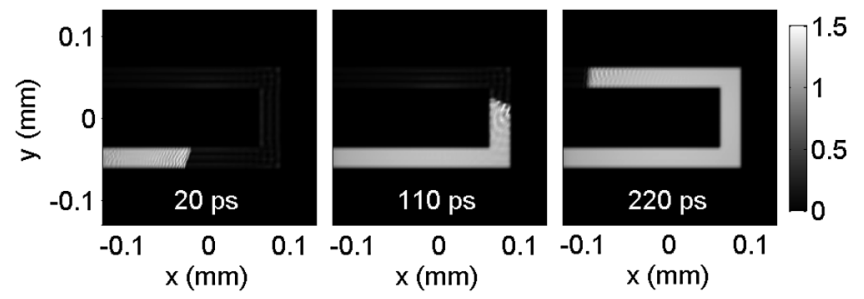

FIG. 2. Spatial polariton intensity profile at different times relative to the pulse arrival time. The polariton lifetime was $\tau=$ $3 \mathrm{ps}$ and the polariton dispersion was calculated with a two oscillator model in which the exciton-photon coupling energy was $3 \mathrm{meV}$ and the photon effective mass was $\times 10^{-5}$ the free electron mass. Both the cw pump and pulse correspond to optical fields at normal incidence; that is, they have Gaussian distributions in reciprocal space centered at zero in-plane wave vector. The pumps are tuned $1 \mathrm{meV}$ above the bare polariton eigenenergy.

analogous to the optical switching waves observed in bistable semiconductor microresonators with fast electronic nonlinearity [47]. The average signal propagation speed depends strongly on the intensity of the driving $\mathrm{cw}$ field. For our parameters it is $1.8 \times 10^{6} \mathrm{~m} / \mathrm{s}$. We believe the signal propagation speed could be enhanced by driving the system with a finite in-plane wave vector; however, this would introduce a dependence of the signal propagation speed on the neuron in-plane direction.

Logic gates. - Logic gates, for creating optical circuits in the microcavity plane, can be created by exploiting the polarization degree of freedom of polaritons. When considering the polarization degree of freedom, our system demonstrates a multistability [24] rather than bistability; both the $\sigma_{+}$and $\sigma_{-}$polariton field intensities can exhibit the $S$-shaped curve of Fig. 1, and both can lie either on the upper or lower branch of the curve.

We now consider the merging of two polariton neurons, by restructuring the potential profile. The system is again excited by a broad but weak Gaussian cw pump, which is now elliptically polarized with a bias towards the $\sigma_{+}$ polarization. The two input channels are independently excited by either $\sigma_{+}$or $\sigma_{-}$pulses. In Fig. 3 we plot the circular polarization degree in real space for the case of oppositely polarized inputs (top row) and the case of two $\sigma_{-}$inputs (bottom row).

In our calculations we assumed $\alpha_{2}=-0.5 \alpha_{1}$. Although in a single quantum well sample a value $\alpha_{2}=-0.1 \alpha_{1}$ has been experimentally inferred [28,37], we expect $\alpha_{2}=$ $-0.5 \alpha_{1}$ to be more realistic in modern microcavities with multiple quantum wells. The small value $\alpha_{2}=$ $-0.1 \alpha_{1}$ in single quantum well samples is explained theoretically from the dominance of the exchange term in the exciton-exciton scattering [36,38] over the Coulomb interaction. However, the exchange interaction is a contact interaction, which requires two electrons or two holes in the same quantum well. In a multiple quantum well struc-
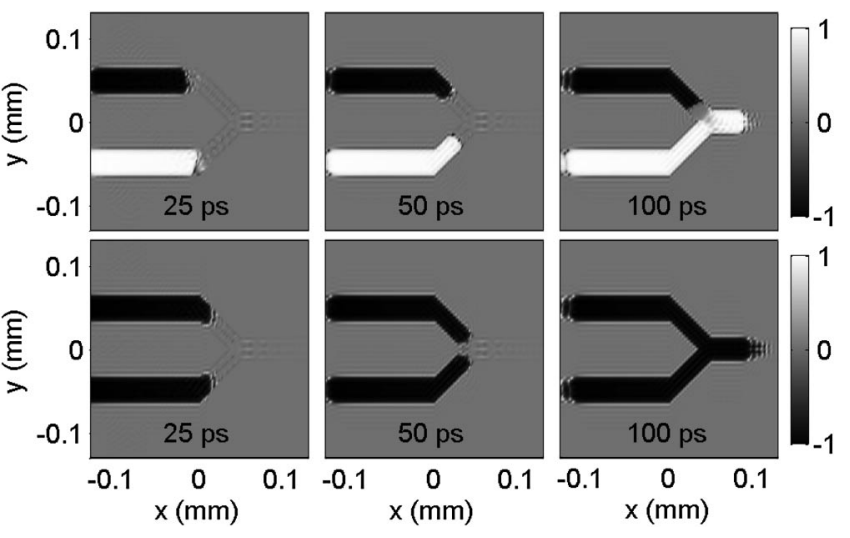

FIG. 3. Circular polarization degree, $\rho_{c}=\frac{\left|\psi_{+}\right|^{2}-\left|\psi_{-}\right|^{2}}{\left|\psi_{+}\right|^{2}+\left|\psi_{-}\right|^{2}}$, in a region where two polariton neurons merge, at different times relative to the pulse arrival time. In the top row the system is excited with oppositely circularly polarized pulses; in the bottom row it is excited with two $\sigma_{-}$polarized pulses.

ture the probability of finding two excitons in the same quantum well is strongly reduced. It is possible to vary the relative strengths of the exchange and Coulomb interactions, and hence the ratio of $\alpha_{2}$ to $\alpha_{1}$, by varying the number of quantum wells in the microcavity design.

When the signals of polariton neurons firing with opposite spin polarization overlap (Fig. 3), we find that only the $\sigma_{+}$signal continues. This is due to the bias of the $\mathrm{cw}$ background field toward $\sigma_{+}$and a negative value of $\alpha_{2}$. This means that the system behaves as an OR logic gate from which the output is $\sigma_{+}$polarized if either input is $\sigma_{+}$ polarized (alternatively we would have an AND gate if the background cw field had opposite $\rho_{c}$ ).

For the parameters used in our device an uncertainty in the arrival time of pulses of at least $10 \mathrm{ps}$ is allowed (note that this is $10 \%$ of the device operation time). The uncertainty can be estimated as the difference in arrival time at the junction of $\sigma_{+}$and $\sigma_{-}$polarized signals when triggered simultaneously. The allowed uncertainty can be increased by adjusting the relative intensities of the cw $\sigma_{+}$ and $\sigma_{-}$components (to alter the propagation speed of $\sigma_{+}$ and $\sigma_{-}$signals) or by increasing the distance between the excitation points and the junction.

The power requirements of the cw optical pump would make up the majority of the power consumption of the device. We note that in Ref. [43] hysteresis was observed in GaAs based microcavities using a power of $2.8 \mathrm{~mW}$. Given that for our parameters the logic gate can be operated at $5 \mathrm{GHz}$ (which allows for a generous $100 \mathrm{ps}$ to reset the device in addition to the $100 \mathrm{ps}$ switching time) and occupies an area of $200 \mu \mathrm{m} \times 200 \mu \mathrm{m}$, the performance characteristics are competitive with recent proposals for optical logic gates [48-52]. Furthermore, in microcavities with a larger exciton-exciton interaction strength (e.g., $\mathrm{GaN}$ based microcavities suitable for room temperature operation) lower power requirements could be achieved. 
We also note that the required pump power is sensitive to the polariton lifetime (or cavity $Q$ factor).

Conclusion. - The short-range propagation of polaritons enables the successive switching of neighboring regions along a multistable channel in space from a low intensity stable state to a high intensity stable state. This switching allows a signal to continue over distances longer than the distance a single polariton could travel before it decays (the distance is limited by the extent of the background cw field, which must have sufficient intensity at a given point for a multistability to exist). When channels merge the polarization of the output propagating signal depends on the ingoing polarizations in a logical way, which allows the construction of binary logic gates. This gives us the opportunity to build optical circuits, in which multiple elements are integrated within a single microcavity structure.

I. A. S. acknowledges the support from the Grant of the President of Russian Federation. T. C. H. L. acknowledges support from the E.P.S.R.C.

[1] V.R. Almedia, C.A. Barrios, R. R. Panepucci, and M. Lipson, Nature (London) 431, 1081 (2004).

[2] G. Khitrova et al., Rev. Mod. Phys. 71, 1591 (1999).

[3] A. V. Kavokin, J. J. Baumberg, G. Malpuech, and F. P. Laussy, Microcavities (Oxford University, New York, 2007).

[4] C. Weisbuch, M. Nishioka, A. Ishikawa, and Y. Arakawa, Phys. Rev. Lett. 69, 3314 (1992).

[5] T. Freixanet, B. Sermage, A. Tiberj, and R. Planel, Phys. Rev. B 61, 7233 (2000).

[6] P. G. Savvidis et al., Phys. Rev. Lett. 84, 1547 (2000).

[7] J. J. Baumberg et al., Phys. Rev. B 62, R16247 (2000).

[8] C. Diederichs et al., Nature (London) 440, 904 (2006).

[9] S. Christopoulos et al., Phys. Rev. Lett. 98, 126405 (2007).

[10] L. V. Butov, Nature (London) 447, 540 (2007).

[11] D. Bajoni et al., Phys. Rev. Lett. 100, 047401 (2008).

[12] J. Kasprzak et al., Nature (London) 443, 409 (2006).

[13] R. Balili et al., Science 316, 1007 (2007).

[14] C. W. Lai et al., Nature (London) 450, 529 (2007).

[15] H. Deng et al., Phys. Rev. Lett. 99, 126403 (2007).

[16] K. G. Lagoudakis et al., arXiv:0801.1916v1.

[17] D. Bajoni et al., Phys. Rev. B 77, 113303 (2008).

[18] A. Amo et al., arXiv:0711.1539v1.
[19] I. A. Shelykh, A. V. Kavokin, and G. Malpuech, Phys. Status Solidi (b) 242, 2271 (2005).

[20] C. Leyder et al., Nature Phys. 3, 628 (2007).

[21] I. Shelykh et al., Phys. Rev. B 70, 035320 (2004).

[22] C. Leyder et al., Phys. Rev. Lett. 99, 196402 (2007).

[23] C.-H. Sun and P. Jiang, Nat. Photon. 2, 9 (2008).

[24] N. A. Gippius et al., Phys. Rev. Lett. 98, 236401 (2007).

[25] R. Idrissi Kaitouni et al., Phys. Rev. B 74, 155311 (2006).

[26] R. B. Balili, D. W. Snoke, L. Pfeiffer, and K. West, Appl. Phys. Lett. 88, 031110 (2006).

[27] W. Langbein et al., Phys. Rev. B 75, 075323 (2007).

[28] I. A. Shelykh et al., Phys. Rev. Lett. 97, 066402 (2006).

[29] I. Carusotto and C. Ciuti, Phys. Rev. Lett. 93, 166401 (2004).

[30] F. Dalfovo, S. Giorgini, L. P. Pitaevskii, and S. Stringari, Rev. Mod. Phys. 71, 463 (1999).

[31] A. J. Leggett, Rev. Mod. Phys. 73, 307 (2001).

[32] L. Pitaevskii and S. Stringari, Bose-Einstein Condensation (Oxford University, New York, 2003).

[33] D. M. Whittaker, Phys. Status Solidi (c) 2, 733 (2005).

[34] D. Sanvitto et al., Phys. Rev. B 73, 241308 (2006).

[35] G. Malpuech et al., Phys. Rev. Lett. 98, 206402 (2007).

[36] C. Ciuti et al., Phys. Rev. B 58, 7926 (1998).

[37] P. Renucci et al., Phys. Rev. B 72, 075317 (2005).

[38] M. Combescot and O. Betbeder-Matibet, Phys. Rev. B 74, 125316 (2006).

[39] S. Schumacher, N. H. Kwong, and R. Binder, Phys. Rev. B 76, 245324 (2007).

[40] Yu. G. Rubo, A. V. Kavokin, and I. A. Shelykh, Phys. Lett. A 358, 227 (2006).

[41] D. N. Krizhanovskii et al., Phys. Rev. B 73, 073303 (2006).

[42] D. M. Whittaker, Phys. Rev. B 71, 115301 (2005).

[43] A. Baas, J. Ph. Karr, H. Eleuch, and E. Giacobino, Phys. Rev. A 69, 023809 (2004).

[44] N. A. Gippius et al., Europhys. Lett. 67, 997 (2004).

[45] M. Wouters and I. Carusotto, Phys. Rev. B 75, 075332 (2007).

[46] I. A. Shelykh, T. C. H. Liew, and A. V. Kavokin, Phys. Rev. Lett. 100, 116401 (2008).

[47] I. Ganne, G. Slekys, I. Sagnes, and R. Kuszelewicz, Phys. Rev. B 63, 075318 (2001).

[48] P. J. Harding et al., Appl. Phys. Lett. 91, 111103 (2007).

[49] S. Perissinotto et al., Appl. Phys. Lett. 91, 191108 (2007).

[50] W. M. Zhu et al., Appl. Phys. Lett. 91, 261106 (2007).

[51] X. Hu et al., Nat. Photon. 2, 185 (2008).

[52] M. Jarrahi et al., Appl. Phys. Lett. 92, 014106 (2008). 